\title{
Looking for Galactic Diffuse Dark Matter in INO-MagICAL Detector
}

\section{Sanjib Kumar Agarwalla*}

Institute of Physics, Sachivalaya Marg, Sainik School Post, Bhubaneswar 751005, India Homi Bhabha National Institute, Training School Complex, Anushakti Nagar, Mumbai 400085, India

E-mail: sanjib@iopb.res.in

\section{Amina Khatun}

Institute of Physics, Sachivalaya Marg, Sainik School Post, Bhubaneswar 751005, India Homi Bhabha National Institute, Training School Complex, Anushakti Nagar, Mumbai 400085,

India

E-mail: amina@iopb.res.in

\section{Ranjan Laha}

Kavli Institute for Particle Astrophysics and Cosmology (KIPAC), Department of Physics, Stanford University, Stanford, CA 94305, USA

SLAC National Accelerator Laboratory, Menlo Park, CA 94025, USA

E-mail: rlaha@stanford.edu

The Weakly Interacting Massive Particle (WIMP) is a popular particle physics candidate for the dark matter (DM). It can annihilate and/or decay to neutrino and antineutrino pair. The proposed 50 kt Magnetized Iron CALorimeter (MagICAL) detector at the India-based Neutrino Observatory (INO) can observe these pairs over the conventional atmospheric neutrino and antineutrino fluxes. If we do not see any excess of events in ten years, then INO-Magical can place competitive limits on self-annihilation cross-section $(\langle\sigma v\rangle)$ and decay lifetime $(\tau)$ of dark matter at 90\% C.L.: $\langle\sigma v\rangle \leq 1.87 \times 10^{-24} \mathrm{~cm}^{3} \mathrm{~s}^{-1}$ and $\tau \geq 4.8 \times 10^{24} \mathrm{~s}$ for $m_{\chi}=10 \mathrm{GeV}$ assuming the NFW as DM density profile.

The 19th International Workshop on Neutrinos from Accelerators-NUFACT 2017

25-30 September, 2017

Uppsala University, Uppsala, Sweden

\footnotetext{
* Speaker.
} 


\section{Introduction}

The existence of mysterious dark matter is confirmed by various astrophysical [1] and cosmological observations [2] through the gravitational interaction. The data collected by Planck satellite reveal that around $26 \%$ of total energy density of the Universe is composed of dark matter [3]. The particle nature of dark matter is completely unknown. However, with the particle having $\sim 100$ $\mathrm{GeV}$ mass and interaction strength of the order of electro-weak coupling, the predicted relic abundance of cold dark matter (CDM) in the Universe matches with its current value. We call these class of dark matter (DM) as Weakly Interacting Massive Particle (WIMP) [4]. If there is non-zero coupling between dark matter and Standard Model (SM) particles, then we can detect dark matter by indirect way. For example, due to annihilation of dark-matter to any SM particles, we can observe an excess of the stable particles, like photons, neutrinos, which are created in the final state of SM particle's decay chain. In this study, we assume that dark matter $(\chi)$ self-annihilate to neutrino and antineutrino pair, and explore the phenomenological consequences in context of the Magnetized Iron CALorimeter (MagICAL) detector proposed by the India-based Neutrino Observatory (INO) [5] project.

The MagICAL is designed to detect atmospheric neutrinos having multi-GeV energy and coming from all possible directions. The main physics aim of this experiment is to determine the neutrino mass ordering using the Earth matter effect and to measure the atmospheric oscillation parameters precisely [6]. In this paper, we show that the MagICAL detector can also play a very important role to look for Galactic diffuse dark matter having mass in the multi-GeV range.

\section{Dark Matter Inputs}

The spherically symmetric dark matter density parameterization is given by

$$
\rho(r)=\frac{\rho_{0}}{\left[\delta+r / r_{s}\right]^{\gamma} \cdot\left[1+\left(r / r_{s}\right)^{\alpha}\right]^{(\beta-\gamma) / \alpha}} .
$$

The parameter $\rho(r)$ denotes the density as a function of distance $r$ from the center of the galaxy, and $r_{s}$ is the scale radius. The shape of the profile is controlled by $\alpha$ and $\beta, \gamma$, and $\delta$. The local dark matter density at the Solar radius $\left(\mathrm{R}_{s c}\right)$ is $\rho_{s c}$. We take $\mathrm{R}_{s c}=8.5 \mathrm{kpc}$. The parameter $\rho_{0}$ is the normalization constant. We produce all the results for two different DM profiles: the NavarroFrenk-White (NFW) profile [7], and the Burkert ${ }^{1}$ profile [9], and associated parameter values are given in table 1 . We assume that dark matter particle $(\chi)$ and its antiparticle $(\bar{\chi})$ annihilate to

\begin{tabular}{|c|c|c|c|}
\hline & $(\alpha, \beta, \gamma, \delta)$ & $\rho_{s c}\left[\mathrm{GeV} \mathrm{cm}^{-3}\right]$ & $r_{s}[\mathrm{kpc}]$ \\
\hline NFW & $(1,3,1,0)$ & 0.471 & 16.1 \\
Burkert & $(2,3,1,1)$ & 0.487 & 9.26 \\
\hline
\end{tabular}

Table 1: The necessary values of parameters related to dark matter profiles are taken from Ref. [10].

\footnotetext{
${ }^{1}$ We do not present the results with the Burkert profile in this write-up. However the results with the Burkert profile are shown in Ref. [8].
} 
produce a neutrino and an antineutrino in the final state with $100 \%$ branching ratio:

$$
\chi+\bar{\chi} \rightarrow v+\bar{v}
$$

The ratio of $v_{e}, v_{\mu}$, and $v_{\tau}$ at the source are assumed to be 1:1:1, which remains unchanged after reaching the Earth surface due to loss of coherence in flight over astrophysical distances. The $v / \bar{v}$ flux of each flavor of per unit energy range per unit solid angle originated from the dark matter particles annihilation is given by

$$
\frac{d^{2} \Phi_{v / \bar{v}}^{a n n}}{d E d \Omega}=\frac{\left\langle\sigma_{A} v\right\rangle}{2} J_{\Delta \Omega}^{a n n} \frac{R_{s c} \rho_{s c}^{2}}{4 \pi m_{\chi}^{2}} \frac{1}{3} \frac{d N^{a n n}}{d E} .
$$

In above, $\left\langle\sigma_{A} v\right\rangle$ is the self-annihilation cross-section and $m_{\chi}$ is mass of DM particles. Integrating the square of dark matter density over the whole sky and then taking average over $4 \pi$ solid angle, we calculate $J_{\Delta \Omega}^{a n n}$, which is obtained as 3.33 for the NFW profile and 1.6 for the Burkert profile. The factor $\frac{1}{2}$ appears as we assume the dark matter particle is same as its own antiparticle. The factor $\frac{1}{3}$ takes care the flavor ratio of $v / \bar{v}$ on the Earth's surface. For the isotropic production of $v$ and $\bar{v}$ at source, $4 \pi$ comes in the denominator. As dark matter is non-relativistic, $v / \bar{v}$ energy spectrum is written as

$$
\frac{d N^{a n n}}{d E}=\delta\left(E_{v / \bar{v}}-m_{\chi}\right)
$$

In case of decay of dark matter through $\chi \rightarrow v+\bar{v}$ channel with $100 \%$ branching ratio (where the final state neutrino can be of any flavor), $v / \bar{v}$ flux can be written as

$$
\frac{d^{2} \Phi_{v / \bar{v}}^{d e c}}{d E d \Omega}=J_{\Delta \Omega}^{d e c} \frac{R_{s c} \rho_{s c}}{4 \pi m_{\chi} \tau} \frac{1}{3} \frac{d N^{d e c}}{d E} .
$$

The factor $\frac{1}{3}$ and $4 \pi$ appear due to the same reasons as described for the annihilation case. The parameter $J_{\Delta \Omega}^{d e c}$ represents the average value of line of sight integration of dark matter density over whole sky. We get $J_{\Delta \Omega}^{d e c}=2.04$ for the NFW profile and 1.85 for the Burkert profile. For decaying dark matter, the energy spectrum of neutrino is given by

$$
\frac{d N^{d e c}}{d E}=\delta\left(E_{v / \bar{v}}-m_{\chi} / 2\right) .
$$

\section{Results}

This section is devoted to show results. In Fig. 1, we present the atmospheric neutrino events integrated over the reconstructed neutrino zenith angle $\cos \theta \in[-1,-0.5]$ using $500 \mathrm{kt} \cdot y \mathrm{r}$ exposure of MagICAL. The black solid and red dashed lines represents the events in absence (ATM) and presence of dark matter $(\mathrm{ATM}+\mathrm{DM})$ respectively. The left panel is for dark matter annihilation and right panel is for decaying dark matter. Here, we assume that dark matter has the mass of $30 \mathrm{GeV}$. In this case, each of the final state $v$ and $\bar{v}$ from the dark matter annihilation (decay) have 30 (15) $\mathrm{GeV}$ energy. Therefore we see an excess of $v_{\mu}$ events around 30 (15) GeV of reconstructed neutrino energy for annihilating (decaying) dark matter in Fig. 1. We estimate the sensitivity of MagICAL 

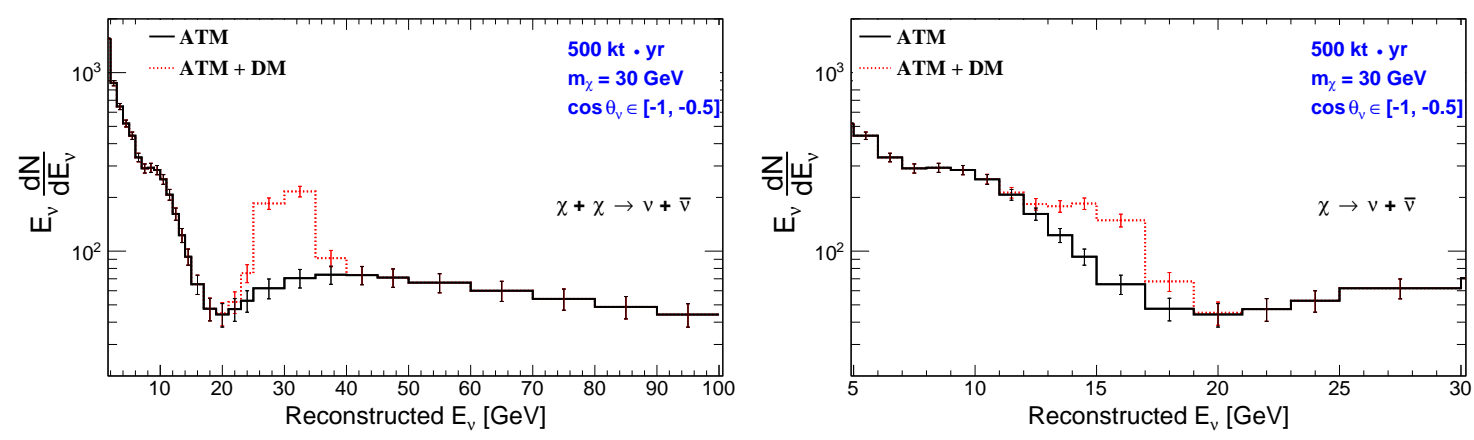

Figure 1: Event spectra of atmospheric $v_{\mu}$ is presented by black solid line. The red dashed lines are for events in presence of annihilation (left panel) and decay (right panel) of dark matter having $30 \mathrm{GeV}$ mass. Both panels are for integrating over $\cos \theta \in[-1,-0.5]$ using $500 \mathrm{kt} \cdot \mathrm{yr}$ exposure of MagICAL. The choice of mass hierarchy is normal ordering (NO).
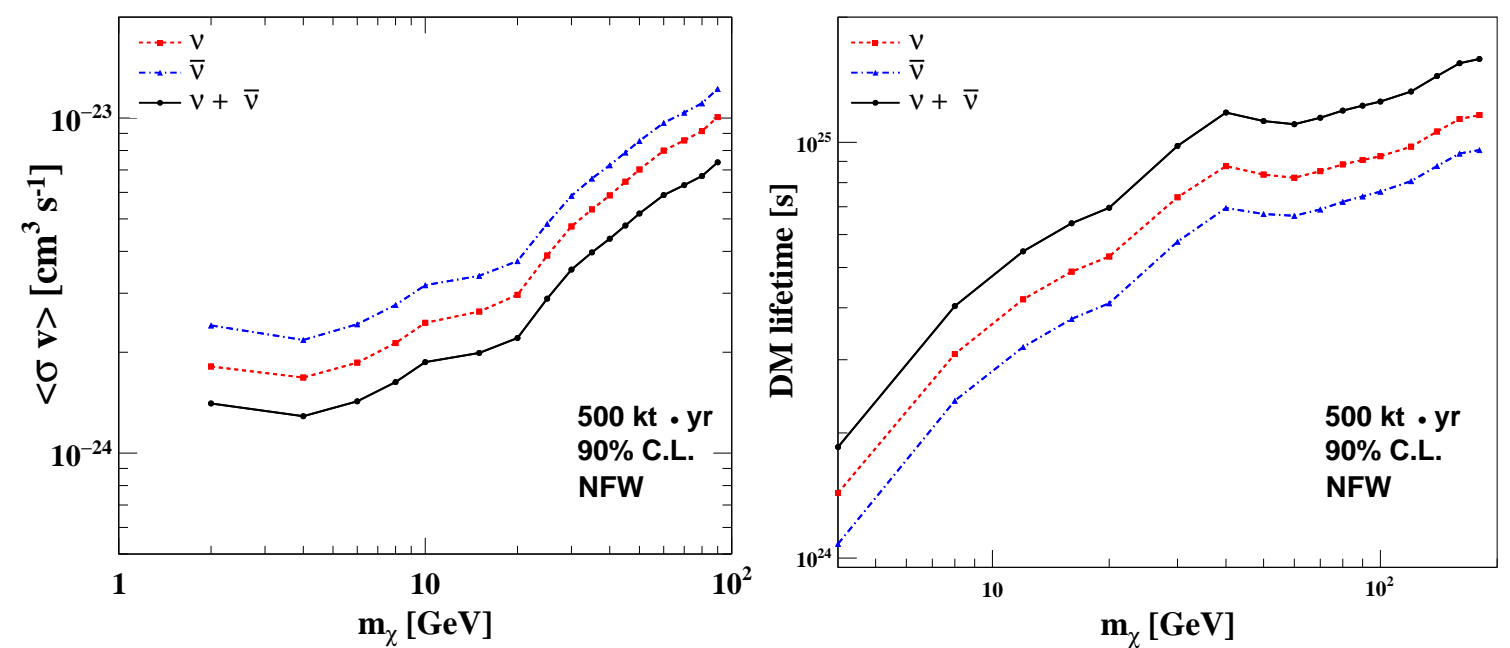

Figure 2: Left panel presents the upper limit on $\langle\sigma v\rangle$ at $90 \%$ C.L. (1 d.o.f.) for the process $\chi \chi \rightarrow v \bar{v}$. The right panel shows the lower bounds on decay lifetime for $\chi \rightarrow v \bar{v}$ at $90 \%$ C.L. (1 d.o.f.). For both the cases, we use $500 \mathrm{kt} \cdot \mathrm{yr}$ exposure of MagICAL, NFW profile, and normal ordering.

to place the upper limits on the self-annihilation cross-section and lower limit on the dark matter decay lifetime. For the details regarding the simulation technique and the discussion on systematic uncertainties, please take a look at the Ref. [8]. Fig. 2 presents the upper limits on self-annihilation cross-section of DM particles for the process $\chi \chi \rightarrow v \bar{v}$ (see left panel) and lower limits on decay lifetime for $\chi \rightarrow v \bar{v}$ process (see right panel) at $90 \%$ C.L. (1 d.o.f.). The red dashed, blue dotdashed, and black solid lines are obtained from only $v_{\mu}$, only $\bar{v}_{\mu}$, and total $v_{\mu}$ and $\bar{v}_{\mu}$ respectively. We use $500 \mathrm{kt} \cdot \mathrm{yr}$ exposure of the MagICAL detector and normal mass ordering (NO). An important point to be noted is that the ability to analyze the neutrino and antineutrino data separately helps 
to explore the processes which involve lepton number violating DM. As we go to higher energies, constraints on the self-annihilation cross-section get deteriorated, and for decay lifetime, bounds get improved. We understand these features using different $m_{\chi}$ dependence of signal to background ratio in case of annihilation and decay of dark matter. For detailed explanation, see Ref. [8].

\subsection{Comparison with other experiments}

There are constraints on the self-annihilation cross-section from the experiments like SuperKamiokande [11, 12], IceCube [10, 13], and ANTARES [14, 15]. In left panel of Fig. 3, we show these limits along with the expected bound from MagICAL with $500 \mathrm{kt} \cdot \mathrm{yr}$ exposure as obtained in this analysis (black solid line). In the multi-GeV energy range, the MagICAL detector is found to place the most stringent constraint than other detectors. We compare the current bound on the dark
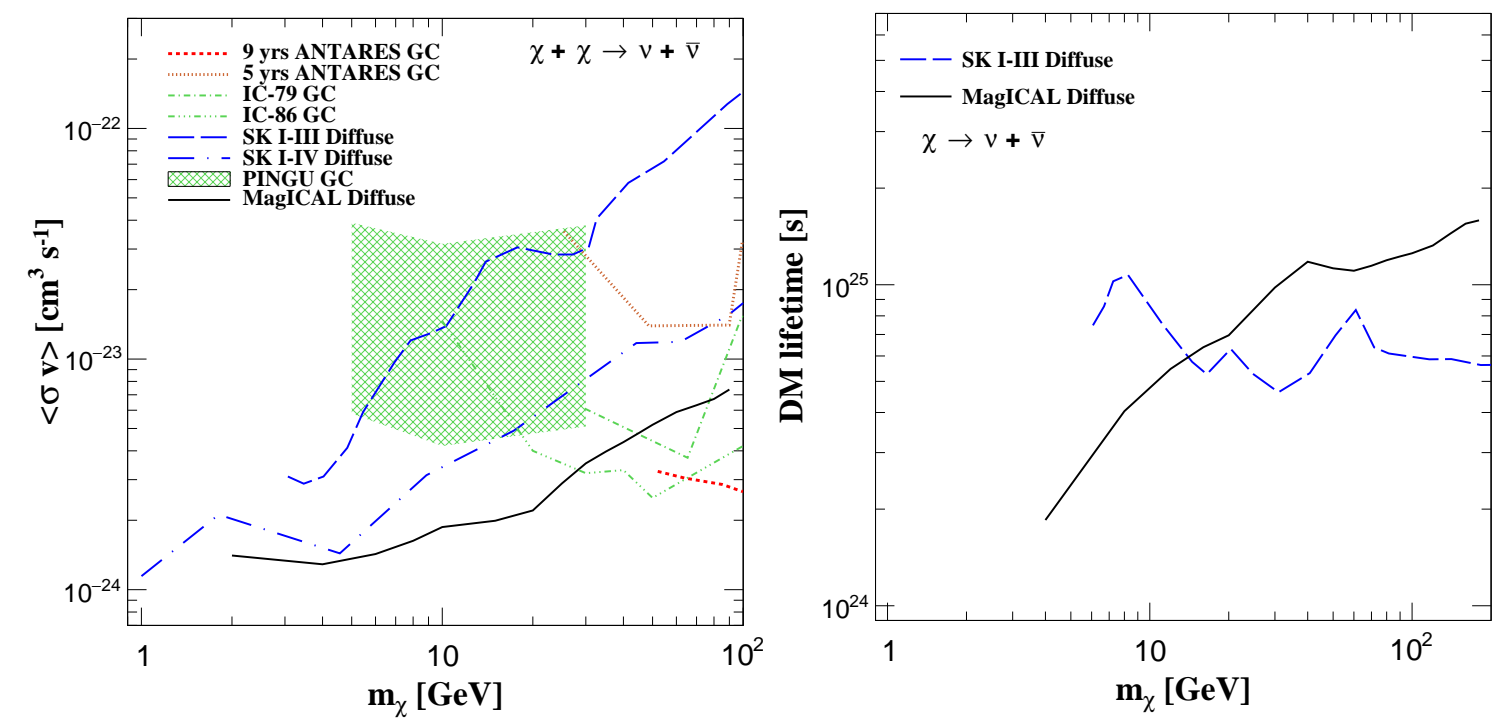

Figure 3: (a) Left panel shows the current bounds on self-annihilation cross-section at $90 \%$ C.L. (1 d.o.f.) obtained using first three phases data of Super-Kamiokande in Ref. [11] (blue long-dashed line), using four phases data of SuperKamiokande in Ref. [12] (blue long-dash-dotted line), using IceCube data in Ref. [10] (green dot-dashed) and Ref. [13] (green triple-dot-dashed lines), and using ANTARES data in Ref. [14] (red dotted) and Ref. [15] (red dashed lines). The future sensitivity of PINGU [16] (green shaded region) with its one year of exposure is also shown. The limits obtained from our analysis using $500 \mathrm{kt} \cdot y \mathrm{r}$ MagICAL is plotted in black solid line. The right panel shows the current bound on decay lifetime of DM at 90\% C.L. (1 d.o.f.) from Super-Kamiokande [11] (blue long-dashed line) and the expected limit from MagICAL (black solid line) using $500 \mathrm{kt} \cdot \mathrm{yr}$ exposure.

matter decay lifetime from the first three phases of Super-Kamiokande data [11] and the expected limit from MagICAL detector in right panel of Fig. 3.

\section{Concluding Remarks}

We study the capability of the INO-MagICAL detector to probe the Galactic diffuse dark matter. The expected limit on the self-annihilation cross-section of dark matter having mass 10 
$\mathrm{GeV}$ is $\langle\sigma v\rangle \leq 1.87 \times 10^{-24} \mathrm{~cm}^{3} \mathrm{~s}^{-1}$ at $90 \%$ C.L. (1 d.o.f.) using $500 \mathrm{kt} \cdot \mathrm{yr}$ exposure of MagICAL and assuming the NFW as dark matter density profile. The ability to distinguish the neutrino and antineutrino in MagICAL gives an opportunities to probe lepton number violating dark matter interactions.

\section{References}

[1] L. E. Strigari, Galactic Searches for Dark Matter, Phys. Rept. 531 (2013) 1-88, [arXiv:1211.7090].

[2] G. Steigman, Neutrinos And Big Bang Nucleosynthesis, Adv. High Energy Phys. 2012 (2012) 268321, [arXiv:1208.0032].

[3] Planck Collaboration, P. A. R. Ade et al., Planck 2015 results. XIII. Cosmological parameters, arXiv: 1502.01589.

[4] G. Jungman, M. Kamionkowski, and K. Griest, Supersymmetric dark matter, Phys. Rept. 267 (1996) 195-373, [hep-ph/9506380].

[5] India-based Neutrino Observatory (INO), http://www.ino.tifr.res.in/ino/.

[6] ICAL Collaboration, S. Ahmed et al., Physics Potential of the ICAL detector at the India-based Neutrino Observatory (INO), arXiv:1505.07380.

[7] J. F. Navarro, C. S. Frenk, and S. D. M. White, The Structure of cold dark matter halos, Astrophys. J. 462 (1996) 563-575, [astro-ph/9508025].

[8] A. Khatun, R. Laha, and S. K. Agarwalla, Indirect searches of Galactic diffuse dark matter in INO-MagICAL detector, JHEP 06 (2017) 057, [arXiv: 1703.10221$].$

[9] A. Burkert and J. Silk, On the structure and nature of dark matter halos, in Dark matter in Astrophysics and Particle Physics (H. V. Klapdor-Kleingrothaus and L. Baudis, eds.), p. 375, 1999. astro-ph/9904159.

[10] IceCube Collaboration, M. G. Aartsen et al., Search for Dark Matter Annihilation in the Galactic Center with IceCube-79, Eur. Phys. J. C75 (2015), no. 10 492, [arXiv: 1505.07259 ].

[11] P. Mijakowski, Direct and Indirect Search for Dark Matter. PhD thesis, Warsaw, Inst. Nucl. Studies, 2011.

[12] Super-Kamiokande Collaboration, P. Mijakowski, Indirect searches for dark matter particles at Super-Kamiokande, J. Phys. Conf. Ser. 718 (2016), no. 4042040.

[13] IceCube Collaboration, M. G. Aartsen et al., Search for Neutrinos from Dark Matter Self-Annihilations in the center of the Milky Way with 3 years of IceCube/DeepCore, arXiv: 1705.08103.

[14] ANTARES Collaboration, S. Adrian-Martinez et al., Search of Dark Matter Annihilation in the Galactic Centre using the ANTARES Neutrino Telescope, JCAP 1510 (2015), no. 10068 , [arXiv:1505.04866].

[15] A. Albert et al., Results from the search for dark matter in the Milky Way with 9 years of data of the ANTARES neutrino telescope, arXiv: 1612.04595.

[16] IceCube PINGU Collaboration, M. G. Aartsen et al., Letter of Intent: The Precision IceCube Next Generation Upgrade (PINGU), arXiv:1401.2046. 